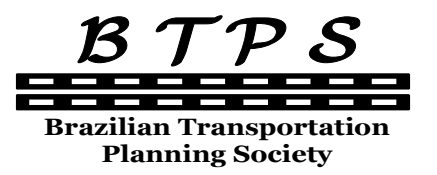

\author{
Journal of Transport Literature \\ Vol. 8, n. 4, pp. 146-163, Oct. 2014 \\ Research Directory
}

JTL | RELIT

www.journal-of-transport-literature.org ISSN 2238-1031

\title{
The effect of air cargo traffic on regional job creation in Turkey
}

[0 efeito do tráfego de carga aérea na geração de empregos na Turquia]

\author{
Ismail Cagri Ozcan* \\ Ministry of Development - Turkey
}

Submitted 31 Jul 2013; received in revised form 13 Nov 2013; accepted 2 Mar 2014

\begin{abstract}
While there is a large body of literature on air passenger traffic's impact on local employment, the interaction between air cargo traffic and local employment remains relatively untouched. This paper tries to fill in the gap by analyzing the impact of air cargo traffic on local employment in 20 Turkish provinces using Census 2000 data. The results of 2-stage least squares estimations show that air cargo traffic stimulates employment in finance, insurance, real estate and business services and increases the total number of administrative and managerial workers and the total number of clerical and related workers while it tends to reduce employment in agriculture, hunting, forestry and fishing activities and the total number of agricultural, animal husbandry, forestry workers, fishermen and hunters.
\end{abstract}

Key words: air cargo traffic, economic contribution, job creation, 2-stage least squares .

\section{Resumo}

Embora exista uma vasta literatura sobre o impacto do tráfego aéreo de passageiros e a geração de emprego, a interação entre o tráfego de carga aérea e o emprego local permanece relativamente intocado. Este trabalho tenta preencher a lacuna, analisando o impacto do tráfego de carga aérea no emprego local em 20 províncias turcas usando dados do Censo de 2000 . Os resultados de uma regressão com método de mínimos quadrados em dois estágios mostram que o tráfego de carga aérea estimula emprego em finanças, seguros, imobiliário e serviços de negócios e aumenta o número total de trabalhadores em administração e gerência e o número total de trabalhadores de escritório e afins, enquanto ele tende a reduzir emprego na agricultura, caça, silvicultura e atividades de pesca e o número total de produtores agrícolas, de criação de animais, trabalhadores florestais, pescadores e caçadores.

Palavras-Chave: tráfego de carga aérea, contribuição econômica, geração de empregos, mínimos quadrados em dois estágios.

*Email: cagriucla@gmail.com.

\section{Recommended Citation}

Ozcan, I. C. (2014) The effect of air cargo traffic on regional job creation in Turkey. Journal of Transport Literature, vol. 8, n. 4, pp. 146-163. 


\section{Introduction}

Today, world trade is more dependent on air cargo services than ever before. Several factors have contributed to this situation. First, firms aiming to reduce inventory costs and to adopt just-in-time production philosophy have been using air cargo logistics as a way of transporting their products in the fastest and most reliable way. Second, in the markets where products' life spans are short, air cargo is the fastest way to place the products to respond to customers' needs and preferences. Third, its declining costs as a result of liberalization and technological progress make air cargo logistics more appealing. And last, as firms shift their production facilities abroad where lower labor costs create a competitive advantage, they rely more on air cargo logistics.

As air cargo services provide fast and reliable logistics opportunities, businesses located at areas with access to an air cargo network are more likely to grow due to their reduced logistics costs. As a result, areas having access to an air cargo network will have a competitive advantage over the ones lacking such accessibility, and it is most likely that the availability of air cargo services will enable regions to create more employment, especially those having industries and occupations more dependent on such services.

The literature consists of the studies on air passenger traffic's impact on economic development, which was mostly proxied by the generated employment [Irwin and Kasarda, 1991; Goetz, 1992; Hewings et al., 1997; Debbage, 1999; Button et al., 1999; Button and Taylor, 2000; Debbage and Delk, 2001; Hakfoort et al., 2001; Brueckner, 2003; Warren, 2008; Alkaabi and Debbage, 2007; Green, 2007; Rasker et al., 2009; Button et al., 2010]. However, the number of studies on the linkage between air cargo traffic and local employment remains limited. Kasarda (1991) noted that, based on analysis of University of North Carolina Business School's Center for Manufacturing Excellence, the proposed global air cargo-industrial complex in North Carolina could create 30,000 direct manufacturing jobs. Oster et al. (1997) estimated that a 1 unit increase in transportation employment, employment at Federal Express in this case, created 2.75 new jobs in Memphis, 2.93 new jobs in Louisville, 3.53 new jobs in Cincinnati, and 0.07 new jobs in Indianapolis. Bowen et al. (2002) analyzed the linkage between air cargo services and development of manufacturing 
plants at Subic Bay, Philippines, and they underlined that the availability of air cargo express services attracted new production plants in the Subic Bay region. Kasarda and Green (2005) revealed that air cargo was related to both gross domestic product (GDP) and GDP per capita and showed that it was possible to estimate with high accuracy both GDP and GDP per capita using air cargo figures. In their study on northern Nevada, Adrangi et al. (2007) documented a strong relation between air cargo shipments at Reno-Tahoe International Airport and the employment in manufacturing, wholesale trade, and retail sectors in the Reno metropolitan statistical area but their results failed to prove a similar relation between air cargo traffic and employment in the finance industry. Using Taiwanese data for the period 1974-2006, Chang and Chang (2009) found that a bi-directional causality between economic growth and air cargo expansion existed.

This paper tries to define the effect of air cargo traffic on the local employment in terms of industries and occupations by using econometric models. To overcome the problem of causality between air cargo traffic and local employment levels, this study employed twostage least-squares (2SLS) estimation. The most significant advantage of this study is that it includes so many occupations and industries through which a wide range of analyses and interpretations can be made. Comparable studies focus on specific sectors (such as manufacturing, wholesale trade, retail and finance, insurance and real estate) or focus on macroeconomic parameters (such as GDP, per capita GDP, and economic growth). In contrast, this paper is able to identify and analyze 16 different groups of occupations and industries, which provides us a large room for conclusions. The results of 2SLS estimations show that air cargo traffic fosters employment in finance, insurance, real estate and business services and increases the total number of (i) administrative and managerial workers and (ii) clerical and related workers. Meanwhile it tends to reduce employment in agriculture, hunting, forestry and fishing activities and the total number of agricultural, animal husbandry, forestry workers, fishermen and hunters. The following section briefly summarizes the current condition of Turkish air cargo industry. Section 3 describes the methodology of the analysis and the data used. Section 4 discusses the results of the analysis. The conclusion includes the summary of the results, the limitations of the study, and the policy implications. 


\section{Turkish air cargo industry at a glance}

As the global trends suggest, the acceleration of Turkish air cargo traffic is higher than that of Turkish GDP. From 1995 to 2010, Turkish air cargo traffic grew about 8.8\% annually while GDP of Turkey increased $3.7 \%$ annually during the same period (Figure 1). According to Consolidated Report of the $10^{\text {th }}$ Transportation Council of Turkey (10 ${ }^{\text {th }}$ Transportation Council of Turkey, 2009; p. 558), it was estimated that the Turkish air cargo traffic would reach 2.56 million tons per year in 2023 (the 2010 traffic was 580,679 tons) which corresponded an annual growth of $12.1 \%$ between 2010 and 2023. Among 15 airlines registered by Directorate General of Turkish Civil Aviation (DGTCA), 3 airlines solely operate air cargo flights while remaining 12 airlines are certified to operate both passenger and air cargo flights. According to 2010 Annual Report of DGTCA (DGTCA, 2011), 26 of the 332 commercial aircrafts belonging to registered Turkish airline companies are air cargo aircrafts.

Figure 1 - Air Cargo Traffic and GDP of Turkey between 1996-2010

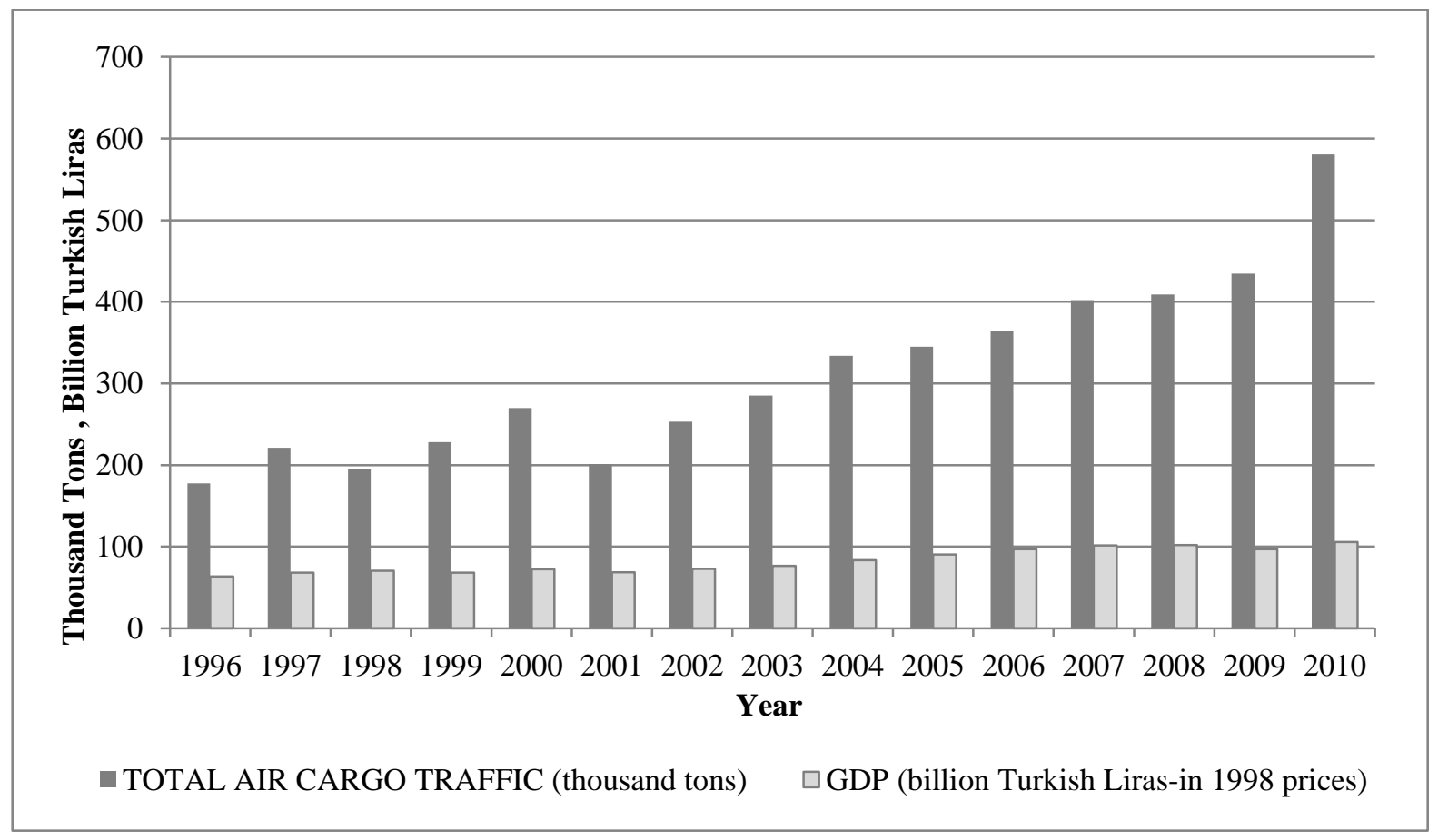

\footnotetext{
${ }^{1}$ Sources: GDP figures come from State Planning Organization's Economic and Social Indicators 1950-2010 (available at www.dpt.gov.tr) and air cargo figures are based on author's calculations.
} 


\title{
2. Methodology and data
}

Air cargo traffic can increase local jobs and local economic activities can stimulate air cargo volume. This chicken-and-egg problem makes econometric estimation of this ambiguous relationship challenging. To overcome this problem of causality, following a methodology similar to that of Brueckner (2003), this study implemented a 2-stage least squares (2SLS) estimation. We used two instruments namely proximity and hub. The suitability of use of proximity as an instrument is rather more unquestionable. The proximity of a province to the closest province having an operating airport with scheduled air service will naturally affect its air cargo traffic and it is very unlikely that the proximity of a province is correlated with the error term of the first stage estimation. Hub, as an instrument, also satisfies the requirement that it should be a determinant of air cargo traffic. However, the use of hub as an instrument is questionable according to Brueckner (2003) because of its possible correlation with the error term of the first stage estimation. Following his explanation for why hub can be employed as an instrument, we can argue that hub can also be used for this study as well since hub locations in Turkey are chosen considering the population of the provinces rather than their air cargo traffics.

The 2-stage least squares (2SLS) estimation is as follows:

\author{
Air cargo traffic $=f(H u b$, Proximity $)$ \\ (first stage) \\ Local employment $=f($ Air cargo traffic, Population, \\ Laborforce, Education, Underdevelopment $)^{2,3}$ \\ (second stage)
}

\footnotetext{
${ }^{2}$ The author has checked for multicollinearity using Variance Inflation Factor (VIF) scores. For the four independent variables, the highest VIF score is 5.80 and the average VIF score is 3.93. To overcome a possible problem of heteroscedasticity, robust standard errors are used in the regression estimations.

${ }^{3}$ The model assumes that provincial borders limit the airports' catchment areas and no passenger leakage exists between the airports of two neighbor provinces.
} 
where

- Air cargo traffic $=$ the five year average of annual total (domestic + international) air cargo traffic handled, in tons, within the airports of each province for the period 19962000.

- $H u b=$ an instrumental dummy variable and equal to 1 for provinces having hub airports (Istanbul and Ankara) in year 2000.

- Proximity $=$ an instrumental variable and equal to the distance, in kilometers, to the closest province having an operating airport with scheduled air service.

- Local employment $=$ the employment figure as of 2000 in each province, which had an airport with air cargo traffic for at least one year during the 5-year period between 1996 and 2000, in terms of one of the 9 industry and 7 occupation classifications.

- Population $=$ the total population at each province in 2000 .

- Laborforce $=$ the percentage of the population between 15 and 64 years old for year 2000.

- Education $=$ the percentage of the population over age 25 with a university degree at each province for year 2000 .

- Underdevelopment $=$ a dummy variable and equal to 1 if the province is classified as "Priority Regions for Development" by the government as of $2000^{4}$.

The best way to set up a linkage between the air cargo traffic and regional employment may be the one which employs the value of air cargo as a proxy for air cargo traffic so that the economic effect of air cargo services can best be analyzed. However, since the statistics of the monetary value of air cargo carried are not available; the weight of air cargo is used as a proxy for the monetary value of the air cargo. Apparently, the weight of air cargo is not the most ideal proxy for the economic value of the air cargo and there is great variation in the values of one pound of wheat and one pound of microprocessors. But as air cargo is preferred for goods having higher value-to-weight ratios, using the weight of the goods carried through air cargo may still work to test the economic contribution of air cargo services.

\footnotetext{
${ }^{4}$ Turkish governments use GDP per capita as the major determinant in classifying Priority Regions for Development. As of 2000, 37 provinces out of 81 were classified as Priority Regions for Development. The average GDP per capita of provinces classified as Priority Regions for Development was $\$ 1,610.4$ while that of those not classified was $\$ 3,014.9$ and the difference in these two figures are significant at $1 \%$ level.
} 
Table 1 - Classification of employment data used as dependent variables in the estimations

\begin{tabular}{|c|c|c|c|}
\hline Industry & Abbreviation & Occupation & Abbreviation \\
\hline $\begin{array}{l}\text { 1. Employment in } \\
\text { agriculture, hunting, } \\
\text { forestry, and fishing }\end{array}$ & $A G R$ & $\begin{array}{l}\text { 1. Total number of scientific, } \\
\text { technical, professional, and } \\
\text { related workers }\end{array}$ & $S C I$ \\
\hline $\begin{array}{l}\text { 2. Employment in mining } \\
\text { and quarrying }\end{array}$ & MINING & $\begin{array}{l}2 . \quad \text { Total number of } \\
\text { administrative and managerial } \\
\text { workers }\end{array}$ & $A D M$ \\
\hline $\begin{array}{l}\text { 3. Employment in } \\
\text { manufacturing industry }\end{array}$ & $M A N U F$ & $\begin{array}{l}\text { 3. Total number of clerical } \\
\text { and related workers }\end{array}$ & $C L E$ \\
\hline $\begin{array}{l}\text { 4. Employment in } \\
\text { electricity, gas, and water }\end{array}$ & $E L E C$ & $\begin{array}{l}\text { 4. Total number of } \\
\text { commercial and sales workers }\end{array}$ & COMMER \\
\hline $\begin{array}{l}\text { 5. Employment in } \\
\text { construction }\end{array}$ & CONS & $\begin{array}{l}\text { 5. Total number of service } \\
\text { workers }\end{array}$ & SERV \\
\hline $\begin{array}{l}\text { 6. Employment in } \\
\text { wholesale and retail trade, } \\
\text { restaurants, and hotels }\end{array}$ & WHOLE & $\begin{array}{l}\text { 6. Total number of } \\
\text { agricultural, animal husbandry, } \\
\text { forestry workers, fishermen, and } \\
\text { hunters }\end{array}$ & $A G R I C U$ \\
\hline $\begin{array}{l}\text { 7. Employment in } \\
\text { transport, communication, } \\
\text { and storage }\end{array}$ & TRANS & $\begin{array}{l}7 . \quad \text { Total number of non- } \\
\text { agricultural production and } \\
\text { related workers, transport } \\
\text { equipment operators, and } \\
\text { laborers }\end{array}$ & NON-AGRICU \\
\hline $\begin{array}{l}\text { 8. Employment in } \\
\text { finance, insurance, real } \\
\text { estate, and business } \\
\text { services }\end{array}$ & FINAN & & \\
\hline $\begin{array}{l}\text { 9. Employment in } \\
\text { community, social, and } \\
\text { personnel services }\end{array}$ & СОММ & & \\
\hline
\end{tabular}

Air cargo traffic figures are measured by the five year average of annual total weight of air cargo handled within the airports of each Turkish province for the period 1996-2000. The original data set included 25 airports from 24 provinces having air cargo traffic for at least one year during the 5-year period between 1996 and 2000. Then airports with quite low air cargo figures were removed from the data set, thus reducing the number of airports analyzed to 21. For Mugla province which has two operating airports within its boundaries, the air 
cargo traffic figures of these two airports were combined to find the total air cargo traffic for that province. As a result, the data set used consisted of observations from 20 provinces (Figure 2).

\section{Figure 2 - The distribution of air cargo traffic among Turkish provinces for $2000^{5}$}

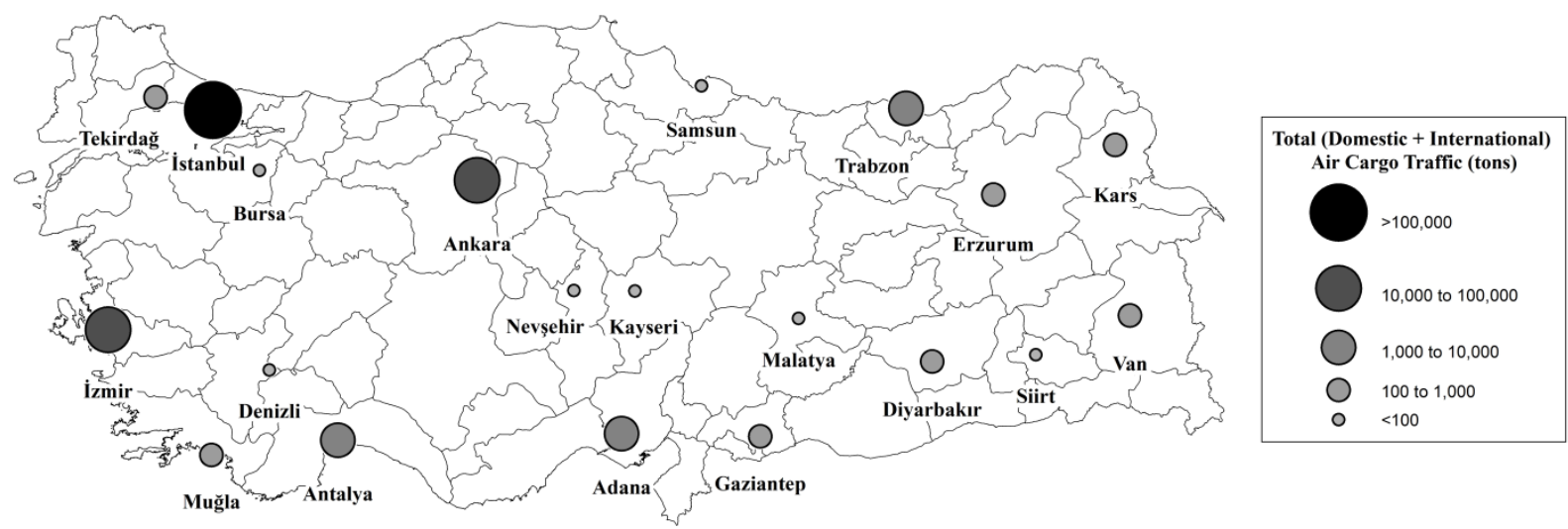

The air cargo traffic figures for the 1996-2000 period are gathered from the statistics yearbook of General Directorate of State Airports Authority (GDSAA) for 2000 (GDSAA, 2001). The online distance calculator of General Directorate of Highways ${ }^{6}$ was used to get the data on proximity. Data regarding employment, population, distribution of population among ages, and educational attainment come from the Turkish Statistical Institute web site (tuikapp.tuik.gov.tr) which provides online statistics of the Census 2000, the last census supplying the detailed data needed. The web site of State Planning Organization ${ }^{7}$ provides the list of provinces classified as "Priority Regions for Development". Table 1 lists 16 employment classifications in terms of industries and occupations that will be used as dependent variables in the estimations and Table 2 reports the summary statistics of the variables used in the estimations.

The focal hypothesis of this study is that air cargo traffic can stimulate local employment in most of the industries and occupations. Therefore it is supposed that air cargo traffic should get a positive coefficient. Similarly, as population is obviously the major determinant of the employment, the independent variable population should get a positive and statistically

\footnotetext{
${ }^{5}$ Prepared by Arda Öcal.

${ }^{6}$ www.kgm.gov.tr.

${ }^{7}$ www.dpt.gov.tr.
} 
significant coefficient. Furthermore, as higher proportions of laborforce will enlarge the pool of the population able to work, it should also get a positive coefficient. Since some industries like FINAN and occupations like SCI and ADM heavily rely on well-educated human resources, it is anticipated that higher ratios of education should stimulate employment especially in these industries and occupations. On the other hand, it is believed that underdeveloped provinces generally fail to attract high value-added employment, such as FINAN, SCI, and ADM which necessitate qualified human resources, and as a result this study expects that being an underdeveloped province should reduce employment in such industries and occupations. The independent dummy variable underdevelopment, which is equal to 1 for less developed provinces, should therefore get a negative coefficient for such industries and occupations.

Table 2 - Summary Statistics

\begin{tabular}{|c|c|c|c|c|}
\hline Variable & Mean & Standard Deviation & Minimum & Maximum \\
\hline Air cargo traffic & $10,913.7$ & $37,819.62$ & 2.2 & 169,974 \\
\hline Hub & 0.1 & 0.31 & 0 & 1 \\
\hline Proximity & 240.3 & 106.75 & 81 & 453 \\
\hline Population & $1,737,031$ & $2,172,406$ & 263,676 & $10,018,735$ \\
\hline Laborforce & 0.63 & 0.06 & 0.50 & 0.70 \\
\hline Education & 0.05 & 0.02 & 0.02 & 0.10 \\
\hline Underdevelopment & 0.45 & 0.51 & 0 & 1 \\
\hline AGR & 215,992 & $87,940.59$ & 44,215 & 377,654 \\
\hline MINING & $1,220.45$ & $1,394.26$ & 24 & 4,906 \\
\hline MANUF & $114,336.4$ & $242,716.9$ & 1,701 & $1,097,051$ \\
\hline ELEC & $2,785.25$ & $4,170.67$ & 277 & 14,968 \\
\hline CONS & $33,270.75$ & $48,048.06$ & 1,985 & 215,925 \\
\hline WHOLE & 83,881 & $144,653.8$ & 3,141 & 650,295 \\
\hline TRANS & $27,070.7$ & $49,149.4$ & 1,706 & 221,298 \\
\hline FINAN & $30,302.8$ & $65,747.93$ & 529 & 283,404 \\
\hline COMMU & $127,233.9$ & $172,585.5$ & 16,453 & 696,033 \\
\hline SCI & $59,043.3$ & $93,561.67$ & 3,863 & 394,578 \\
\hline ADM & $12,344.95$ & $22,229.96$ & 477 & 92,038 \\
\hline CLE & $52,247.4$ & $96,422.88$ & 2,566 & 417,970 \\
\hline COMMER & $53,970.6$ & $100,266.1$ & 2,069 & 452,964 \\
\hline SERV & 58,559 & $88,044.83$ & 6,766 & 389,654 \\
\hline AGRICU & $216,641.5$ & $88,384.06$ & 44,254 & 379,385 \\
\hline NON-AGRICU & $183,783.8$ & $316,105.2$ & 14,131 & $1,434,470$ \\
\hline & & & & \\
\hline
\end{tabular}


Table 3 - Regression Results for the second stage of 2SLS estimations for industries ${ }^{8}$

\begin{tabular}{|c|c|c|c|c|c|c|c|c|c|c|c|c|c|c|c|c|c|c|}
\hline $\begin{array}{l}\text { Indep. } \\
\text { Vars }\end{array}$ & $\begin{array}{l}\text { AGR } \\
\text { (OLS) }\end{array}$ & $\begin{array}{c}\text { AGR } \\
(2 S L S)\end{array}$ & $\begin{array}{l}\text { MINING } \\
\text { (OLS) }\end{array}$ & $\begin{array}{l}\text { MINING } \\
\text { (2SLS) }\end{array}$ & $\begin{array}{l}\text { MANUF } \\
\text { (OLS) }\end{array}$ & $\begin{array}{l}\text { MANUF } \\
\text { (2SLS) }\end{array}$ & $\begin{array}{l}\text { ELEC } \\
(\mathrm{OLS})\end{array}$ & $\begin{array}{l}\text { ELEC } \\
(2 S L S)\end{array}$ & $\begin{array}{l}\text { CONS } \\
\text { (OLS) }\end{array}$ & $\begin{array}{l}\text { CONS } \\
\text { (2SLS) }\end{array}$ & $\begin{array}{c}\text { WHOLE } \\
\text { (OLS) }\end{array}$ & $\begin{array}{l}\text { WHOLE } \\
(2 S L S)\end{array}$ & $\begin{array}{l}\text { TRANS } \\
\text { (OLS) }\end{array}$ & $\begin{array}{l}\text { TRANS } \\
(2 S L S)\end{array}$ & $\begin{array}{c}\text { FINAN } \\
\text { (OLS) }\end{array}$ & $\begin{array}{l}\text { FINAN } \\
\text { (2SLS) }\end{array}$ & $\begin{array}{l}\text { COMMU } \\
\text { (OLS) }\end{array}$ & $\begin{array}{l}\text { COMMU } \\
\text { (2SLS) }\end{array}$ \\
\hline Intercept & $\begin{array}{l}2.229 \\
(0.65)\end{array}$ & $\begin{array}{l}1.354 \\
(0.44)\end{array}$ & $\begin{array}{c}-12.188^{* * * *} \\
(3.08)\end{array}$ & $\begin{array}{c}-10.758^{* *} \\
(2.35)\end{array}$ & $\begin{array}{c}-15.684 * * * \\
(5.77)\end{array}$ & $\begin{array}{c}-14.964 * * * \\
(4.95)\end{array}$ & $\begin{array}{c}-4.944^{*} \\
(2.13)\end{array}$ & $\begin{array}{l}-4.165 \\
(1.64)\end{array}$ & $\begin{array}{c}-6.165^{* * *} \\
(4.47)\end{array}$ & $\begin{array}{c}-5.768 * * * \\
(4.18)\end{array}$ & $\begin{array}{c}-7.629 * * * \\
(5.42)\end{array}$ & $\begin{array}{c}-7.626 * * * \\
(5.10)\end{array}$ & $\begin{array}{c}-6.687 * * * * \\
(5.05)\end{array}$ & $\begin{array}{c}-6.360^{* * *} \\
(5.11)\end{array}$ & $\begin{array}{c}-11.732 * * * \\
(15.87)\end{array}$ & $\begin{array}{c}-11.312 * * * \\
(23.80)\end{array}$ & $\begin{array}{l}0.671 \\
(0.62)\end{array}$ & $\begin{array}{l}0.688 \\
(0.66)\end{array}$ \\
\hline $\begin{array}{l}\text { Air cargo } \\
\text { traffic }\end{array}$ & $\begin{array}{l}-0.017 \\
(0.47)\end{array}$ & $\begin{array}{c}-0.119^{*} \\
(1.79)\end{array}$ & $\begin{array}{c}-0.161^{* *} \\
(2.59)\end{array}$ & $\begin{array}{l}0.041 \\
(0.29)\end{array}$ & $\begin{array}{c}-0.062^{*} \\
(1.95)\end{array}$ & $\begin{array}{l}0.036 \\
(0.52)\end{array}$ & $\begin{array}{l}-0.033 \\
(1.29)\end{array}$ & $\begin{array}{l}0.067 \\
(1.09)\end{array}$ & $\begin{array}{l}0008 \\
(0.37)\end{array}$ & $\begin{array}{l}0.054 \\
(1.54)\end{array}$ & $\begin{array}{l}-0.011 \\
(0.47)\end{array}$ & $\begin{array}{l}-0.009 \\
(0.22)\end{array}$ & $\begin{array}{l}0.001 \\
(0.07)\end{array}$ & $\begin{array}{l}0.040 \\
(1.22)\end{array}$ & $\begin{array}{l}-0.000 \\
(0.02)\end{array}$ & $\begin{array}{c}0.050^{\text {*** }} \\
(2.91)\end{array}$ & $\begin{array}{l}0.028 \\
(1.18)\end{array}$ & $\begin{array}{l}0.025 \\
(0.67)\end{array}$ \\
\hline Population & $\begin{array}{c}0.526 * * * \\
(3.09)\end{array}$ & $\begin{array}{c}0678 * * * * \\
(4.82)\end{array}$ & $\begin{array}{c}0.839^{* * * *} \\
(3.22)\end{array}$ & $\begin{array}{l}0.539 \\
(1.44)\end{array}$ & $\begin{array}{c}1.492 * * * \\
(9.32)\end{array}$ & $\begin{array}{c}1.346^{* * * *} \\
(6.54)\end{array}$ & $\begin{array}{c}0.904 * * * \\
(5.24)\end{array}$ & $\begin{array}{c}0.756 \text { *** } \\
(4.00)\end{array}$ & $\begin{array}{c}1.016 \text { *** } \\
(16.37)\end{array}$ & $\begin{array}{c}0.947 * * * \\
(13.06)\end{array}$ & $\begin{array}{c}1.076 * * * \\
(11.67)\end{array}$ & $\begin{array}{c}1.073 * * * \\
(9.70)\end{array}$ & $\begin{array}{c}1.021^{* * * *} \\
(12.78)\end{array}$ & $\begin{array}{c}0.963 * * * \\
(10.68)\end{array}$ & $\begin{array}{c}1.223 * * * \\
(28.12)\end{array}$ & $\begin{array}{c}1.148 * * * \\
(25.09)\end{array}$ & $\begin{array}{c}0.811^{* * * *} \\
(11.00)\end{array}$ & $\begin{array}{c}0.815^{* * * *} \\
(8.49)\end{array}$ \\
\hline Laborforce & $\begin{array}{l}4.526 \\
(1.47)\end{array}$ & $\begin{array}{l}2.990 \\
(0.89)\end{array}$ & $\begin{array}{c}11.539 * * * \\
(4.44)\end{array}$ & $\begin{array}{c}15.192 \text { *** } \\
(4.59)\end{array}$ & $\begin{array}{c}11.657^{* * * *} \\
(4.21)\end{array}$ & $\begin{array}{c}13.380 * * * \\
(4.54)\end{array}$ & $\begin{array}{l}-1.740 \\
(1.42)\end{array}$ & $\begin{array}{l}-0.081 \\
(0.05)\end{array}$ & $\begin{array}{l}2.958^{*} \\
(1.99)\end{array}$ & $\begin{array}{c}3.656^{* * *} \\
(2.61)\end{array}$ & $\begin{array}{c}5.064 * * * \\
(3.58)\end{array}$ & $\begin{array}{c}5.138 * * * \\
(3.65)\end{array}$ & $\begin{array}{c}2.526^{* * *} \\
(2.30)\end{array}$ & $\begin{array}{c}3.130^{* *} \\
(2.68)\end{array}$ & $\begin{array}{c}5.388^{* * * *} \\
(7.60)\end{array}$ & $\begin{array}{c}6.176^{* * * *} \\
(8.12)\end{array}$ & $\begin{array}{c}-2.348^{*} \\
(1.80)\end{array}$ & $\begin{array}{c}-2.484 * \\
(1.78)\end{array}$ \\
\hline Education & $\begin{array}{l}-6.449 \\
(0.68)\end{array}$ & $\begin{array}{l}-0.465 \\
(0.05)\end{array}$ & $\begin{array}{l}13.163 \\
(1.15)\end{array}$ & $\begin{array}{l}-3.069 \\
(0.19)\end{array}$ & $\begin{array}{c}-28.014 * * \\
(2.63)\end{array}$ & $\begin{array}{c}-35.527 * * * \\
(3.15)\end{array}$ & $\begin{array}{c}21.128 * * \\
(2.88)\end{array}$ & $\begin{array}{l}14.154 \\
(1.72)\end{array}$ & $\begin{array}{l}-0.137 \\
(0.04)\end{array}$ & $\begin{array}{l}-2.854 \\
(0.74)\end{array}$ & $\begin{array}{l}2.945 \\
(0.43)\end{array}$ & $\begin{array}{l}2.536 \\
(0.39)\end{array}$ & $\begin{array}{l}7.397 \\
(1.66)\end{array}$ & $\begin{array}{l}4.991 \\
(1.13)\end{array}$ & $\begin{array}{c}9.921 * * * \\
(3.54)\end{array}$ & $\begin{array}{c}6.764 * * \\
(2.94)\end{array}$ & $\begin{array}{c}13.449 * * * \\
(3.19)\end{array}$ & $\begin{array}{c}14.274 * * * \\
\quad(3.26)\end{array}$ \\
\hline $\begin{array}{l}\text { Under } \\
\text { develop. }\end{array}$ & $\begin{array}{c}0.303^{*} \\
(1.81)\end{array}$ & $\begin{array}{c}0.404^{* * *} \\
(2.38)\end{array}$ & $\begin{array}{l}-0.368 \\
(0.87)\end{array}$ & $\begin{array}{l}-0.322 \\
(0.67)\end{array}$ & $\begin{array}{c}-1.008^{* * * *} \\
(5.23)\end{array}$ & $\begin{array}{c}-1.007 * * * \\
(4.68)\end{array}$ & $\begin{array}{l}-0.097 \\
(0.50)\end{array}$ & $\begin{array}{r}-0.133 \\
(0.63)\end{array}$ & $\begin{array}{l}-0.223 \\
(1.54)\end{array}$ & $\begin{array}{c}-0.269^{*} \\
(1.95)\end{array}$ & $\begin{array}{c}-0.305^{* *} \\
(2.42)\end{array}$ & $\begin{array}{c}-0.293^{*} \\
(2.12)\end{array}$ & $\begin{array}{l}-0.124 \\
(1.19)\end{array}$ & $\begin{array}{l}-0.157 \\
(1.51)\end{array}$ & $\begin{array}{c}-0.164^{* * *} \\
(2.18)\end{array}$ & $\begin{array}{c}-0.203^{* *} \\
(2.65)\end{array}$ & $\begin{array}{l}-0.005 \\
(0.05)\end{array}$ & $\begin{array}{l}-0.038 \\
(0.39)\end{array}$ \\
\hline $\mathrm{R}^{2}$ & 0.658 & 0.713 & 0.855 & 0.822 & 0.961 & 0.957 & 0.921 & 0.922 & 0.980 & 0.983 & 0.979 & 0.979 & 0.984 & 0.985 & 0.995 & 0.997 & 0.976 & 0.974 \\
\hline
\end{tabular}

\footnotetext{
${ }^{8}$ Notes: (1) Air cargo traffic, Population, and all employment figures of industries in natural logs, (2) t-statistics in parenthesis based on robust regressions, (3) *, $* *$ and $* * *$ stand for significance levels at $1 \%, 5 \%$, and $10 \%$, respectively, (4) number of observations $=20$.

JTL-RELIT | Journal of Transport Literature, Manaus, vol. 8, n. 4, Oct. (2014)
} 
Table 4 - Regression Results for the second stage of 2SLS estimations for occupations ${ }^{9}$

\begin{tabular}{|c|c|c|c|c|c|c|c|c|c|c|c|c|c|c|}
\hline & $\begin{array}{c}\text { SCI } \\
(\mathrm{OLS})\end{array}$ & $\begin{array}{c}\text { SCI } \\
(2 \mathrm{SLS})\end{array}$ & $\begin{array}{l}\text { ADM } \\
(\mathrm{OLS})\end{array}$ & $\begin{array}{c}\text { ADM } \\
(2 S L S)\end{array}$ & $\begin{array}{l}\text { CLE } \\
(\mathrm{OLS})\end{array}$ & $\begin{array}{l}\text { CLE } \\
(2 \mathrm{SLS})\end{array}$ & $\begin{array}{l}\text { COMME } \\
\text { (OLS) }\end{array}$ & $\begin{array}{l}\text { COMME } \\
\text { (2SLS) }\end{array}$ & $\begin{array}{l}\text { SERV } \\
\text { (OLS) }\end{array}$ & $\begin{array}{l}\text { SERV } \\
\text { (2SLS) }\end{array}$ & $\begin{array}{l}\text { AGRICU } \\
(\text { OLS })\end{array}$ & $\begin{array}{l}\text { AGRICU } \\
\text { (2SLS) }\end{array}$ & $\begin{array}{l}\text { NON-AGRICU } \\
\text { (OLS) }\end{array}$ & $\begin{array}{l}\text { NON- } \\
\text { AGRICU } \\
\text { (2SLS) }\end{array}$ \\
\hline Intercept & $\begin{array}{c}-6.601 * * * \\
(14.54)\end{array}$ & $\begin{array}{c}-6.525 * * * \\
(12.50)\end{array}$ & $\begin{array}{c}-9.348 * * * \\
(8.06)\end{array}$ & $\begin{array}{c}-8.496 \text { **** } \\
(6.40)\end{array}$ & $\begin{array}{c}-7.400 * * * * \\
(16.51)\end{array}$ & $\begin{array}{c}-7.097 * * * \\
(19.51)\end{array}$ & $\begin{array}{c}-9.969 * * * \\
(14.37)\end{array}$ & $\begin{array}{c}-9.856 * * * * \\
(14.65)\end{array}$ & $\begin{array}{l}0.760 \\
(0.35)\end{array}$ & $\begin{array}{l}0.701 \\
(0.31)\end{array}$ & $\begin{array}{l}2.220 \\
(0.65)\end{array}$ & $\begin{array}{l}1.360 \\
(0.44)\end{array}$ & $\begin{array}{c}-4.459 * * \\
(2.54)\end{array}$ & $\begin{array}{c}-4.062 * * \\
(2.38)\end{array}$ \\
\hline Air cargo traffic & $\begin{array}{l}-0.010 \\
(1.56)\end{array}$ & $\begin{array}{l}0.001 \\
(0.06)\end{array}$ & $\begin{array}{c}-0.043 * \\
(2.04)\end{array}$ & $\begin{array}{c}0.067^{*} \\
(2.03)\end{array}$ & $\begin{array}{l}-0.003 \\
(0.39)\end{array}$ & $\begin{array}{c}0.034 * * * \\
(3.04)\end{array}$ & $\begin{array}{l}-0.010 \\
(0.69)\end{array}$ & $\begin{array}{l}0.005 \\
(0.29)\end{array}$ & $\begin{array}{l}0.006 \\
(0.20)\end{array}$ & $\begin{array}{l}-0.002 \\
(0.03)\end{array}$ & $\begin{array}{l}-0.017 \\
(0.48)\end{array}$ & $\begin{array}{c}-0.117 * \\
(1.77)\end{array}$ & $\begin{array}{l}0.017 \\
(0.50)\end{array}$ & $\begin{array}{l}0.061 \\
(1.16)\end{array}$ \\
\hline Population & $\begin{array}{c}1.052 * * * \\
(51.71)\end{array}$ & $\begin{array}{c}1.035^{* * *} \\
(36.44)\end{array}$ & $\begin{array}{c}1.123 * * * \\
(15.58)\end{array}$ & $\begin{array}{c}0.959 * * * \\
(9.89)\end{array}$ & $\begin{array}{c}1.163 * * * \\
(40.08)\end{array}$ & $\begin{array}{c}1.108 * * * \\
(43.26)\end{array}$ & $\begin{array}{c}1.219 * * * \\
(25.63)\end{array}$ & $\begin{array}{c}1.196^{* * * *} \\
(23.84)\end{array}$ & $\begin{array}{c}0.792 * * * \\
(7.18)\end{array}$ & $\begin{array}{c}0.804 * * * \\
(6.56)\end{array}$ & $\begin{array}{l}0.528 * * * \\
(3.12)\end{array}$ & $\begin{array}{c}0.677^{* * * *} \\
(4.84)\end{array}$ & $\begin{array}{c}0.990^{* * * *} \\
(9.55)\end{array}$ & $\begin{array}{c}0.925^{* * * *} \\
(7.50)\end{array}$ \\
\hline Laborforce & $\begin{array}{c}2.861 * * * \\
(5.68)\end{array}$ & $\begin{array}{c}3.067 * * * \\
(5.55)\end{array}$ & $\begin{array}{c}3.395^{* * *} \\
(2.69)\end{array}$ & $\begin{array}{c}5.253 * * * \\
(3.88)\end{array}$ & $\begin{array}{c}1.309^{* *} \\
(2.32)\end{array}$ & $\begin{array}{c}1.895^{* * * *} \\
(4.30)\end{array}$ & $\begin{array}{c}5.248 * * * \\
(8.57)\end{array}$ & $\begin{array}{c}5.521 * * * \\
(6.99)\end{array}$ & $\begin{array}{l}-3.397 \\
(1.52)\end{array}$ & $\begin{array}{l}-3.547 \\
(1.66)\end{array}$ & $\begin{array}{l}4.490 \\
(1.46)\end{array}$ & $\begin{array}{l}2.983 \\
(0.90)\end{array}$ & $\begin{array}{c}4.077 * * \\
(2.32)\end{array}$ & $\begin{array}{c}4.710^{* * *} \\
(2.46)\end{array}$ \\
\hline Education & $\begin{array}{c}9.575 * * * \\
(5.26)\end{array}$ & $\begin{array}{c}8.643 * * * \\
(4.39)\end{array}$ & $\begin{array}{l}9.691 \\
(1.67)\end{array}$ & $\begin{array}{l}1.814 \\
(0.33)\end{array}$ & $\begin{array}{l}9.182 * * * \\
(4.63)\end{array}$ & $\begin{array}{c}6.801 * * * \\
(4.23)\end{array}$ & $\begin{array}{l}-3.096 \\
(1.71)\end{array}$ & $\begin{array}{c}-4.295^{*} \\
(1.91)\end{array}$ & $\begin{array}{c}18.607 * \\
(2.10)\end{array}$ & $\begin{array}{c}19.272 * * \\
(2.20)\end{array}$ & $\begin{array}{l}-6.246 \\
(0.67)\end{array}$ & $\begin{array}{r}-0.379 \\
(0.04)\end{array}$ & $\begin{array}{l}-8.421 \\
(1.30)\end{array}$ & $\begin{array}{c}-10.782 \\
(1.75)\end{array}$ \\
\hline Underdevelopment & $\begin{array}{c}0.064 * \\
(1.80)\end{array}$ & $\begin{array}{c}0.068^{*} \\
(2.03)\end{array}$ & $\begin{array}{c}-0.313 * * \\
(2.97)\end{array}$ & $\begin{array}{c}-0.344 * * * \\
(3.61)\end{array}$ & $\begin{array}{c}-0.069^{*} \\
(2.08)\end{array}$ & $\begin{array}{c}-0.094 * * * \\
(3.08)\end{array}$ & $\begin{array}{c}-0.216^{* *} \\
(2.70)\end{array}$ & $\begin{array}{c}-0.215^{* * *} \\
(2.60)\end{array}$ & $\begin{array}{c}-0.345^{*} \\
(2.03)\end{array}$ & $\begin{array}{c}-0.347^{*} \\
(1.94)\end{array}$ & $\begin{array}{l}0.303^{*} \\
(1.82)\end{array}$ & $\begin{array}{c}0.403 * * \\
(2.38)\end{array}$ & $\begin{array}{c}-0.552 * * * \\
(5.05)\end{array}$ & $\begin{array}{c}-0.608 * * * \\
(5.65)\end{array}$ \\
\hline $\mathrm{R}^{2}$ & 0.961 & 0.998 & 0.985 & 0.985 & 0.997 & 0.998 & 0.995 & 0.995 & 0.953 & 0.952 & 0.664 & 0.717 & 0.970 & 0.972 \\
\hline
\end{tabular}

\footnotetext{
${ }^{9}$ Notes: (1) Air cargo traffic, Population, and all employment figures of industries in natural logs, (2) t-statistics in parenthesis based on robust regressions, (3) *, $* *$ and $* * *$ stand for significance levels at $1 \%, 5 \%$, and $10 \%$, respectively, (4) number of observations $=20$.

JTL-RELIT | Journal of Transport Literature, Manaus, vol. 8, n. 4, Oct. (2014)
} 
Table 5 - Scenario analysis for a $10 \%$ increase in air cargo traffic ${ }^{10}$

\begin{tabular}{|c|c|c|c|c|c|}
\hline & & $\begin{array}{l}\text { Air cargo traffic } \\
\text { (tons) }\end{array}$ & AGR & FINAN & $\begin{array}{c}\text { Net change in total employment for } \\
\text { industries }\end{array}$ \\
\hline \multirow{3}{*}{$\begin{array}{l}\text { PANEL } \\
\text { A }\end{array}$} & Median province & 202.0 & $220,054.5$ & $7,973.5$ & n.a. \\
\hline & $\begin{array}{l}\text { Change in the employment after } \\
10 \% \text { increase in air cargo traffic }\end{array}$ & n.a. & $-2,618.7$ & 39.9 & $-2,578.8$ \\
\hline & $\begin{array}{l}\text { Final figures for median province } \\
\text { after } 10 \% \text { increase in air cargo traffic }\end{array}$ & 222.2 & $219,792.6$ & $7,977.5$ & $-2,578.8$ \\
\hline
\end{tabular}

\begin{tabular}{|c|c|c|c|c|c|c|}
\hline & & $\begin{array}{l}\text { Air cargo traffic } \\
\text { (tons) }\end{array}$ & $\mathrm{ADM}$ & CLE & AGRICU & $\begin{array}{l}\text { Net change in total employment for } \\
\text { occupations }\end{array}$ \\
\hline \multirow{3}{*}{$\begin{array}{c}\text { PANEL } \\
\text { B }\end{array}$} & Median province & 202.0 & $4,374.0$ & $17,878.5$ & $221,939.5$ & n.a. \\
\hline & $\begin{array}{l}\text { Change in the employment after } \\
10 \% \text { increase in air cargo traffic }\end{array}$ & n.a. & 29.3 & 60,8 & $-2,596.7$ & $-2,506.6$ \\
\hline & $\begin{array}{l}\text { Final figures for median province } \\
\text { after } 10 \% \text { increase in air cargo traffic }\end{array}$ & 222.2 & $4,376.9$ & $17,884.6$ & $221,679.8$ & $-2,506.6$ \\
\hline
\end{tabular}

${ }^{10}$ n.a. $=$ not applicable.

JTL-RELIT | Journal of Transport Literature, Manaus, vol. 8, n. 4, Oct. (2014) 


\section{Empirical results}

The regression results for the first stage of 2 SLS estimation $\left(R^{2}=0.482\right)$ are as follows:

$$
\text { Air cargo traffic }=4.419 \text { hub }+0.009 \text { proximity }+3.145
$$

where air cargo traffic figures are in natural logs and the t-statistics (respectively 3.91***; 1.76* and $2.76^{* *}$ ) are based on robust standard errors. The $*, * *$ and $* * *$ stand for significance levels at $1 \%, 5 \%$, and 10\%, respectively. Table 3 and Table 4 present the results of 2SLS estimations for the effect of air cargo traffic on employment in individual industries and occupations, respectively. According to Table 3, a $1 \%$ increase in air cargo traffic leads to $0.05 \%$ increase in the employment in finance, insurance, real estate, and business services (FINAN) while such an increase in air cargo traffic reduces employment in agriculture, hunting, forestry and fishing $(A G R)$ by $0.119 \%$. The results reported in Table 4 reveal that a when air cargo traffic increases by $1 \%$, the total number of administrative and managerial workers $(A D M)$ increases by $0.067 \%$ and the total number clerical and related workers (CLE) increases by $0.034 \%$ while the same increase in air cargo traffic tends to reduce agricultural, animal husbandry, forestry workers, fishermen and hunters (AGRICU) by $0.117 \%$.

To put it in a more concrete way, take a hypothetical province (let us call it median province) having the median values of the dependent and independent variables. Table 5 shows the values of Air cargo traffic, AGR, FINAN, ADM, CLE, and AGRICU for the median province. Panel A of Table 5 reports the possible changes in AGR, FINAN when Air cargo traffic increase by $10 \%$ and Panel B of Table 5 shows the comparable changes in ADM, CLE, and AGRICU when Air cargo traffic increase by $10 \%$.

When air cargo traffic goes from 202 tons to 222.2 tons (or increases by $10 \%$ ), holding other variables of the model constant, the employment in FINAN, ADM, and CLE increase by 39.9, 29.3, and 60.8, respectively while the employment in $A G R$ and $A G R I C U$ decrease by 2,618.7 and 2,596.7, respectively. While air cargo growth stimulates employment in FINAN, ADM, and CLE, it significantly decreases agricultural employment, both in terms of industry $(A G R)$ and 
occupation $(A G R I C U)$. After a $10 \%$ increase in Air cargo traffic, the net change in total employment will be around $-2,579$ in terms of industries and -2,507 in terms of occupations.

One major implication of the findings presented in Table 3 and Table 4 is that air cargo traffic fosters employment in many industries and occupations including a high-paid and high valueadded occupation like administrative and managerial workers (ADM), implying that air cargo services help attract qualified human resources ${ }^{11}$. Another striking result of the regression analysis is the negative relation between air cargo traffic and agricultural jobs in terms of both agriculture industry $(A G R)$ and agricultural occupations $(A G R I C U)$. One possible explanation of this finding may be the disguised unemployment concentrated in agricultural activities. Limited alternative employment opportunities and the below-average educational and skill levels of the human resources at the rural regions stimulate excessive employment at the family-owned agricultural enterprises. With the increase in air cargo traffic, which is a proxy of the development of economic activities that mean more and alternative (and most probably betterpaid) jobs, the disguised unemployment in agricultural occupations is expected to shift to other better-paid jobs.

Table 3 and 4 also include interesting findings about the control variables used in the estimations. As one can easily expect, population had positive coefficients, statistically significant at $1 \%$ level, in all of the 16 regressions but the one for MINING proving that the population of a province is the major factor determining the employment level at that province. Similarly, again in parallel with the previous expectation, Laborforce, which is a proxy for the percentage of the population able to work, has positive and statistically significant coefficients in 6 of the 9 estimations for industries (Table 3) and in 5 of the 7 estimations for occupations (Table 4). Regarding educational attainment, 2SLS estimations produced quite mixed results. The employment in FINAN, COMMU, SCI, CLE, and SER increases with increasing percentage of the population over age 25 with a university degree while higher educational attainment tends to decrease employment in MANUF and COMME. Finally, underdevelopment, which controls the regional disparities, had statistically significant and negative coefficients for MANUF, CONS, WHOLE, FINAN, ADM, CLE, COMME, SERV, and NON-AGRICU implying that the

\footnotetext{
${ }^{11}$ Income statistics of Turkish Statistical Institute for years 2006 and 2010 reveal that management is the highest earning occupation class in Turkey (tuik.gov.tr).
} 
employment in these industries and occupations tend to decrease at underdeveloped provinces. On the other hand, Table 3 and 4 also show that being an underdeveloped province increases employment in AGR, SCI, and AGRICU. A comment then can be made, which is similar to that of the linkage between air cargo traffic and employment in both $A G R$ and $A G R I C U$, on these results. It is believed that low-income provinces may more depend on agriculture industry which is generally associated with low-income and unqualified employment. However, this study fails to explain why being an underdeveloped province increases employment in SCI.

\section{Conclusion}

This paper attempts to determine the linkage between air cargo traffic and its possible effects on local employment in Turkey in terms of industries and occupations. The limited number of observations may limit the possibility for making policy implications. However, the boom in the Turkish air transport industry within the last decade increased the number of provinces having air cargo traffic to 38 in 2011. Using the data from a higher number of airports and those data that will be gathered through upcoming census, more conclusive implications can be derived. The findings here suggest that air cargo traffic fosters local employment in finance, insurance, real estate and business services (FINAN), number of administrative and managerial workers (ADM) and the total number clerical and related workers (CLE) while it tends to reduce employment in agriculture, hunting, forestry and fishing $(A G R)$ and the total number of non-agricultural production and related workers, transport equipment operators, and laborers (AGRICU).

Policy implications are important. Given the stimulus effect of air cargo traffic on value-added and well-paid occupations and industries, governments should take measures to improve it. First, capacity constraints should be improved by adding new capacity where needed, especially at the hub airports. The proposed new air cargo terminal at Istanbul Ataturk International Airport, which handled almost $85 \%$ of all Turkish air cargo traffic in 2011, will increase the air cargo handling capacity enormously and help enhance quicker logistic services. GDSAA has been authorized to launch the project through the Build-Operate-Transfer scheme and pre-tender studies have been continuing since 2009. Second, ongoing airport master plan studies tendered by GDSAA should include planning for future air cargo facilities and necessary land should be 
allocated to air cargo facilities, especially at those airports facing difficulties with finding additional land adjacent to airport sites. In addition, subsidized rates for the rental of land and warehouse space may be offered to private air cargo companies at small airports where profitable operations are hard to achieve due to low air cargo volumes. This is quite important considering how air cargo traffic can be essential in creating additional employment in small provinces where new employment opportunities are limited. Third, multimodal transportation infrastructure should be enhanced so that airports can be well connected with the rest of the transportation network that will in turn enable more effective and efficient intermodal freight transportation. Last but not least, custom services should be improved and new custom directorates should be established at airports lacking them to speed up the logistics processes and to enable air cargo shipments through direct international flights without the need for a connecting flight to an airport with custom facilities.

The other side of the medal, however, includes a significant reduction in agricultural employment, both in terms of industry $(A G R)$ and occupation $(A G R I C U)$. According to the analyses presented here, a 10\% increase in Air cargo traffic would decrease agricultural employment around 2,619 in terms of industries $(A G R)$ and around 2,697 in terms of occupations $(A G R I C U)$. In addition, regression results also reveal that the net employment change in the total economy is expected to be negative after an increase in Air cargo traffic and this finding, whose validity is deeply questioned, is against the general belief. The author believes that the Air cargo traffic must stimulate the overall economy and the shift of disguised employment in the agricultural activities into alternative well-paid jobs can be the underlying reason for the employment reduction in agricultural activities. The author also argues that Air cargo traffic stimulates employment in some other industry and occupation classifications as well. Since the limited number of observations makes us fail to document such an econometric relationship, further work needs to clarify this linkage using the data both of the higher number of airports and of the upcoming census. But this study is still noteworthy since it sheds light on the effect of air cargo traffic on employment in individual industries and occupations. 


\section{References}

$10^{\text {th }}$ Transportation Council of Turkey (2009) Consolidated Report of 10th Transportation Council of Turkey.

Adrangi, B., Gritta, R. D. and Raffiee, K. (2007) Air cargo shipments and regional employment: the northern Nevada case. Journal of Business \& Economics Research, vol. 5, n. 2, pp. 27-44.

Alkaabi, K. A. and Debbage, K. G. (2007) Air passenger demand and skilled labor markets by US metropolitan area. Journal of Air Transport Management, vol. 13, n. 3, pp. 121-130.

Brueckner, J. K. (2003) Airline traffic and urban economic development. Urban Studies, vol. 40, n. 8, pp. $1455-1469$.

Button, K., Lall, S., Stough, R. and Trice, M. (1999) High-technology employment and hub airports. Journal of Air Transport Management, vol. 5, n. 1, pp. 53-59.

Button, K. and Taylor, S. (2000) International air transportation and economic development. Journal of Air Transport Management, vol. 6, n. 4, pp. 209-222.

Button, K., Doh, S. and Yuan, J. (2010) The role of small airports in economic development. Airport Management, vol. 4, n. 2, pp. 125-136.

Chang, Y. H. and Chang, Y. W. (2009) Air cargo expansion and economic growth: Finding the empirical link. Journal of Air Transport Management, vol. 15, n. 5, pp. 264-265.

Debbage, K. G. (1999) Air transportation and urban-economic restructuring: competitive advantage in the US Carolinas. Journal of Air Transport Management, vol. 5, n. 4, pp. 211-221.

Debbage, K. G. and Delk, D. (2001) The geography of air passenger volume and local employment patterns by US metropolitan core area: 1973-1996. Journal of Air Transport Management, vol. 7, n. 3, pp. 159-167.

Directorate General of Turkish Civil Aviation. (2011) 2010 Annual Report of Directorate General of Turkish Civil Aviation, Ankara.

General Directorate of Highways. Online Distance Calculator. Available at www.kgm.gov.tr.

General Directorate of State Airports Authority. (2001) Statistics Yearbook of General Directorate of State Airports Authority for 2000, Ankara.

Goetz, A. R. (1992) Air passenger transportation and growth in the U.S. urban system, 1950-1987. Growth and Change, vol. 23, n. 2, pp. 217-238.

Green, R. K. (2007) Airports and economic development. Real Estate Economics, vol. 35, n. 1, pp. 91112.

Hakfoort, J., Poot, T. and Rietveld, P. (2001) The regional economic impact of an airport: The case of Amsterdam Schiphol Airport. Regional Studies, vol. 35, n. 7, pp. 595-604.

Hewings, G. J. D., Schindler, G. R. and Israilevich, P. R. (1997) Infrastructure and economic development: airport capacity in the Chicago Metropolitan Region, 2001-2018. Journal of Infrastructure Systems, vol. 3, n. 3, pp. 96-102.

Irwin, M. D. and Kasarda, J. D. (1991) Air passenger linkages and employment growth in U.S. metropolitan areas. American Sociological Review, vol. 56, n. 4, pp. 424-537. 
Kasarda, J. D. (1991) Global Air Cargo-Industrial Complexes as Development Tools. Economic Development Quarterly, vol. 5, n. 3, pp. 187-196.

Kasarda, J. D. and Green, J. D. (2005) Air cargo as an economic development engine: A note on opportunities and constraints. Journal of Air Transport Management, vol. 11, n. 6, pp. 459-462.

Oster, C. V., Rubin, B. M. and Strong, J. S. (1997) Economic impacts of transportation investments: the case of Federal Express. Transportation Journal, vol. 37, n. 2, pp. 34-44.

Rasker, R., Gude, P. H., Gude, J. A. and Noort, J. V. D. (2009) The economic importance of air travel in high-amenity rural areas. Journal of Rural Studies, vol. 25, n. 3, pp. 343-353.

State Planning Organization, Available at www.dpt.gov.tr.

State Planning Organization. Economic and social indicators 1950-2010. Available at www.dpt.gov.tr.

Turkish Statistical Institute. Census 2000. Available at tuikapp.tuik.gov.tr.

Turkish Statistical Institute. Available at tuik.gov.tr.

Warren, D. E., (2008) The regional economic effects of commercial passenger air service at small airports. Dissertation, University of Illinois at Urbana-Champaign Department of Agricultural and Consumer Economics. 